\title{
Accumulation of triple recessive alleles for three antinutritional proteins in soybean with black seed coat and green cotyledon
}

\author{
Gyung Young Kang $\cdot$ Sang Woo Choi $\cdot$ Won Gi Chae $\cdot$ Jong Il Chung
}

Received: 27 April 2020 / Revised: 11 May 2020 / Accepted: 12 May 2020

(C) Korean Society for Plant Biotechnology

\begin{abstract}
The black seed coat of soybeans contain anthocyanins which promote health. However, mature soybean seeds contain anti-nutritional factors like lipoxygenase, lectin and Kunitz Trypsin Inhibitor (KTI) proteins. Furthermore, these seeds can be used only after the genetic elimination of these proteins. Therefore, the objective of this study was to develop novel soybean genotypes with black seed coat and triple recessive alleles (lx1lx1lx2lx2lx3lx3, titilele) for lipoxygenase, lectin, and KTI proteins. From a cross of parent1 (lx1lx2lx3/lxllx2lx3, ti/ti, Le/Le) and parent2 (lx1lx2lx3/lx1lx2lx3, Ti/Ti, le/le), $132 \mathrm{~F}_{2}$ seeds were obtained. A 3:1 segregation ratio was observed during F2 seed generation for the inheritance of lectin and KTI proteins. Between a cross of the $L e$ and $T i$ genes, the observed independent inheritance ratio in the $\mathrm{F}_{2}$ seed generation was 9: $3: 3: 1$ (69 Le_Ti_: 32 leleTi_: 22 Le_titi: 9 leletiti $)\left(\chi^{2}=\right.$ $2.87, \mathrm{P}=0.5-0.1)$. From nine $\mathrm{F}_{2}$ seeds with triple recessive

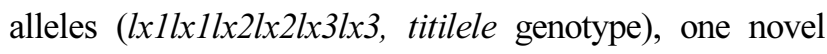
strain posessing black seed coat, and free of lipoxygenase, lectin and KTI proteins, was selected. The seed coat color of the new strain was black and the cotyledon color of the mature seed was green. The weight of 100 seeds belonging to the new strain was $35.4 \mathrm{~g}$. This black soybean strain with lx1lx1lx2lx2lx3lx3, titilele genotype is a novel strain free of lipoxygenase, lectin, and KTI proteins.
\end{abstract}

Keywords Lipoxygenase, Kunitz Trypsin Inhibitor, lectin, lx1lx1lx2lx2lx3lx3 titilele genotype

G. Y. Kang $\cdot$ S. W. Choi $\cdot$ W. G. Chae $\cdot$ J. I. Chung $(\bowtie)$ Department of Agronomy, Gyeongsang National University, JinJu, Korea, 52828

e-mail: jongil@gnu.ac.kr

\section{Introduction}

Soybean [Glycine $\max (\mathrm{L}$.$) Merr.] protein is excellent nu-$ tritional factors and is widely used for human and animal feed in the world. In seed coat of black soybean, anthocyanins are especially abundant (Choung et al. 2001). Anthocyanins from soybean with black seed coat are known to have many pharmaceutical effect. Health-promoting effects such as reduction in the risk of coronary heart disease, regulation of adhesion molecules, protection from reperfusion, and potential antioxidant effects were reported (Burns et al. 2000; Kim et al. 2006). However, a few antinutritional factors such as lipoxygenase, lectin, and Kunitz trypsin inhibitor (KTI) proteins in raw mature soybean seed with black seed coat are present. These components reduce the nutritional value.

Lipoxygenases are a class of enzymes that catalyze the hydroperoxidation of polyunsaturated fatty acids such as the linoleic and linolenic acids. Lipoxygenase proteins constitute about $1 \sim 2 \%$ of the total protein. End-products from lipoxygenase activity are converted to many volatile compounds, which are responsible for the beany flavor in soybean products. Many researchers (Davies and Nielsen, 1986; Hildebrand and Hymowitz, 1981; Kitamura et al. 1983) have reported on the heredity and genetic elimination of lipoxygenase protein. Single dominant genes $(L x 1, L x 2$ and $L x 3)$ control lipoxygenase protein and recessive alleles ( $l x l$, $l \times 2, l \times 3)$ are responsible for absence of lipoxygenase protein in mature seed. Kobayashi et al. (1995) reported that soybean seeds with lipoxygenase free are better accepted due to production of very low levels of hexanal compounds. Macleod and Ames (1988) reported that extra costs need to inactivate lipoxygenase activity by heat at industrial level and the solubility and functionality of proteins was adversely affected. Breeding of soybean cultivar with lipoxygenase free through genetic elimination is the key to get rid of the beany flavour. So far, several cultivars with lipoxygenase free have been 
developed (Chung, 2009; Kim et al. 1997).

Kunitz (1945) isolated and crystallized Kunitz Trypsin Inhibitor (KTI) protein from soybean seeds for the first time. KTI protein is a small and non-glycosylated protein possessing 181 amino acid residues with $21.5 \mathrm{kDa}$. Trypsin is strongly inhibited by KTI protein and food intake is reduced by diminishing digestion and absorption. Four forms of $T i^{a}, T i^{b}, T i^{c}$, and $T i^{d}$ have been reported at a single locus with a codominant multiple allelic series (Orf and Hymowitz, 1979; Hymowitz and Hadley, 1972; Sing et al. 1969). The fifth form as a recessive allele designated $t i$ does not exhibit a soybean KTI protein (Orf and Hymowitz, 1979). Crude protein from titi genotype soybean had a 30 to $50 \%$ reduction in trypsin inhibitor activity compared with 'Amsoy 71' that has the TiTi genotype. The $T i$ gene has been located on chromosome 8 (molecular linkage group A2) of the USDA/Iowa State University soybean linkage map (Cregan et al. 1999; Hildebrand et al. 1980; Kiang, 1987).

Soybean agglutinin (lectin) protein is a major antinutritional element and can strongly endure degradation by proteases under in vitro and in vivo conditions. Soybean lectin protein is a glycoprotein that specifically binds galactose or $\mathrm{N}$-acetylgalactosamine. Molecular weight of soybean lectin protein is $120 \mathrm{kDa}$ (Pull et al. 1978). Soybean lectin protein contains four subunits that each have a molecular weight of $30 \mathrm{kDa}$. The concentration of lectins in soybean seed was ranged $1 \sim 2 \%$ on seed dry mass (George et al. 2008). By proper heating, the biological activity of soybean lectin protein can be reduced. However, considerable quantity is found after heating. The nutritional quality of the soybean protein was affected negatively by this residual soybean lectin and the digestion and absorption of nutrients was decreased (Schulze et al. 1995). Soybean seed lectin was controlled by a single gene designated $L e$ (le) and lele genotype results in the lack of lectin in mature seed (Orf et al. 1978). Several researchers observed that $L e$ and $T i$ loci were independently inherited (Lee et al. 2008; Moraes et al. 2006; Orf and Hymowitz, 1979). The soybean line with triple null recessive genotypes (ti/ti-le/ le-p34/p34) was developed (Schmidt et al. 2015).

Presence of lipoxygenase, lectin, and KTI proteins in mature raw soybean seeds requires heating step to reduce the activity of these antinutritional components. But, excessive heat treatment may diminish amino acid availability. The genetic elimination of these factors could be an alternative to the heat treatment. New black soybean cultivars with free of lipoxygenase, lectin, and KTI proteins improve the nutrition values and food processing properties of soybeans. This cultivar enhances the utilization of soybean in food as well as feed uses. The objective of this study was to improve new black soybean genotype with green cotyledon and triple recessive alleles (lx1lx1lx2lx2lx3lx3titilele) for lipoxygenase, lectin, and KTI proteins. This is the first report on black soybean line with green cotyledon and lx1lx1lx2lx2lx3lx3titilele genotype (free of lipoxygenase, lectin, and KTI proteins).

\section{Materials and Methods}

Genetic population

Four parents ("Gaechuck\#1", "Jimpum\#2", 12N1, and Le-16) were used to create genetic population. Genotype of "Gaechuck\#1" is Lx1Lx1lx2lx2lx3lx3titiLeLe (lipoxygenase-2,3 and KTI proteins free and lectin protein present). "Jinpum\#2" has lx1lx1lx2lx2lx3lx3TiTiLeLe genotype (lipoxygenase-1,2,3 protein free, KTI and lectin proteins present). $12 \mathrm{~N} 1$ parent has $1 \times 1 l \times 1 l x 2 l \times 2 l \times 3 l \times 3$ TiTiLeLe genotype (lipoxygenase-1,2,3 protein free, KTI and lectin proteins present). Le-16 parent has Lx1Lx1Lx2Lx2Lx3Lx3TiTilele genotype (absence of lectin protein and presence of lipoxygenase-1,2,3 and KTI proteins). Color of seed coat, presence or absence for lipoxygenase, lectin, and KTI proteins of four parents are presented in Table 1.

$F_{1}$ seeds were obtained from cross of "Gaechuck\#1" and "Jinpum\#2" parents and were planted in the greenhouse. $F_{2}$ seeds were harvested from $F_{1}$ hybrid plants. From $F_{2}$ seeds, new parent1 with $l \times 1 l x 1 l \times 2 l \times 2 l \times 3 l \times 3$ titiLeLe genotype (lipoxygenase-1,2,3 and KTI proteins free, lectin protein present) was developed. From the cross of $12 \mathrm{~N} 1$ and Le-16 parents, the $F_{1}$ seeds were obtained. $F_{1}$ seeds obtained were planted in the greenhouse. $F_{2}$ seeds from $F_{1}$ hybrid plants

Table 1 Seed coat color, presence or absence of lipoxygenase, lectin, and Kunitz Trypsin Inhibitor (KTI) proteins in four parent strains

\begin{tabular}{ccccc}
\hline Parents & Seed coat color & Lipoxygenase & KTI & Lectin \\
\hline Gaechuck\#1 & Black & 2,3 absence & Absence & Presence \\
Jinpum\#2 & Yellow & $1,2,3$ absence & Presence & Presence \\
12N1 & Black & $1,2,3$ absence & Presence & Presence \\
Le-16 & Yellow & $1,2,3$ presence & Presence & Absence \\
\hline
\end{tabular}


were obtained. From $\mathrm{F}_{2}$ seeds, new parent2 with $l x 1 l x 1$ lx2lx2lx3lx3TiTilele genotype (lipoxygenase-1,2,3 and lectin proteins free, KTI protein present) was developed. From the cross of new parent 1 and new parent $2, F_{1}$ seeds were obtained and were planted in greenhouse. $F_{2}$ seeds were harvested from $F_{1}$ plants. The $F_{2}$ seeds harvested from $F_{1}$ hybrid plants were used to screen the seed with lx1lx1lx2 lx2lx3lx3titilele genotype (lipoxygenase, lectin, and KTI proteins free).

Identification of lipoxygenase protein by SDS-PAGE

Total proteins from the parents, individual $\mathrm{F}_{2}$ seed, and random $\mathrm{F}_{4}$ seeds were obtained to identify the presence ('+') or absence ('-') of lipoxygenase protein. A part of cotyledon from the parent, each $\mathrm{F}_{2}$ seed, and random $\mathrm{F}_{4}$ seed was removed and was incubated for $30 \mathrm{~min}$ in $1 \mathrm{ml}$ Tris-HCl, $\mathrm{pH} 8.0$ and $1.56 \% \mathrm{v} / \mathrm{v}$ ß-mercaptoethanol. Through centrifugation, $50 \mu \mathrm{l}$ of the supernatant was added to an equivalent amount of $5 \mathrm{X}$ sample buffer containing $1 \mathrm{M}$ Tris-HCl, $\mathrm{pH} 6.8,50 \% \mathrm{v} / \mathrm{v}$ glycerol, $1.96 \%$ v/v $\beta$-mercaptoethanol, and $10 \% \mathrm{w} / \mathrm{v}$ sodium dodecyl sulfate (SDS). Sample obtained was boiled at $97^{\circ} \mathrm{C}$ for 5 min and sample was centrifuged. $2 \mu \mathrm{l}$ of the supernatant was loaded on a $12 \%$ acrylamide SDS polyacrylamide gel electrophoresis medium gels in Owl Separation Systems Inc (Model: P9DS, Portsmouth, NH USA). After electrophoresis for $7 \mathrm{hrs}$ at $120 \mathrm{~V}$, gels were stained. For several hours, the gels were destained in destaining solution. Protein marker (Sigma MarkerTM, Product Code: M4038, St. Louis MO USA) was used to identify the presence or absence of lipoxygenase protein (97 kDa).

Identification of lectin and KTI proteins by western blot analysis

Total proteins obtained from parental seeds, individual $\mathrm{F}_{2}$ seed, and random $\mathrm{F}_{4}$ seeds were separated by $10 \%$ or $12 \%$ SDS-PAGE, and transferred onto Immobilon-P membrane (PVDF, Millipore). After blocking for $2 \mathrm{hr}$ in TBS buffer containing $0.1 \%$ Tween $20,20 \mathrm{mM}$ Tris (pH 7.5), 150 $\mathrm{mM} \mathrm{NaCl}$, and $5 \%$ nonfat dried milk (Carnation, Glendale, CA), the membrane were incubated with antibody of KTI and lectin protein for $1 \mathrm{hr}$. The blot was incubated with a horseradish peroxidase conjugated secondary antibody after washing in TBS buffer. Using enhanced chemiluminescence kit (Amersham, Bucking- hamshire, UK), the complex was visualized. Presence or absence of KTI and lectin protein was determined visually. The ratio of segre-

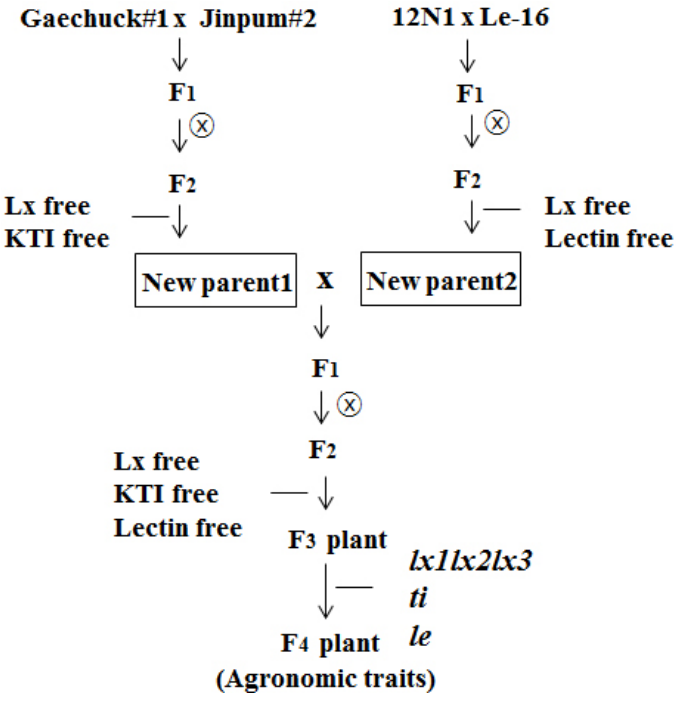

Fig. 1 Schematic representation of the crossover experiment for the development of a novel soybean strain possessing black seed coat, green cotyledon, and lx1lx1lx2lx2lx3lx3, titilele genotype (indicating absence of lipoxygenase, lectin, and KTI proteins)

gation for presence or absence of lectin and KTI proteins was determined by Chi-square analysis.

Improvement of soybean new strain with triple recessive and black seed coat

From the cross of new parent 1 and new parent $2, F_{2}$ seeds were obtained and were used to select the seed with lx1lx1lx2lx2lx3lx3titilele genotype (absence of lipoxygenase, lectin, and KTI proteins). The $\mathrm{F}_{2}$ seeds with triple null alleles (lx1lx1lx2lx2lx3lx3titilele) were planted to advance $F_{2}$ plant generation. Each $F_{2}$ plant with green cotyledon color and black seed coat color was harvested. $F_{3}$ seeds with triple null alleles (lx1lx1lx2lx2lx3lx3titilele) were planted to advance $F_{3}$ plant generation. Each $F_{3}$ plant with a proper agronomical traits was harvested. Random $\mathrm{F}_{4}$ seeds obtained from $\mathrm{F}_{3}$ plants were used to confirm the absence of lipoxygenase, lectin, and KTI proteins. Color of seed coat, hilum, and cotyledon was recorded on $\mathrm{F}_{5}$ seed. Seed weight ( $\mathrm{g} / 100$ seeds) was recorded on the $\mathrm{F}_{4}$ plant generation. Scheme for improvement of lx1lx1lx2lx2lx3lx3titilele genotype (absence of lipoxygenase, lectin, and KTI proteins is presented in Figure 1.

\section{Results}

From the cross of new parent1 (lx1lx2lx3/lx1lx2lx3, ti/ti, Le/Le) and new parent2 (lxllx2lx3/lxllx2lx3, Ti/Ti, le/le), $132 F_{2}$ seeds were obtained. Genotype of $F_{1}$ seeds was 
Table 2 Heredity pattern for the presence or absence of lectin and KTI proteins in the $F_{2}$ seed generation

\begin{tabular}{|c|c|c|c|c|c|}
\hline \multicolumn{2}{|c|}{ Seed protein } & \multicolumn{2}{|c|}{ Number of seed } & \multirow{2}{*}{$\begin{array}{l}\chi^{2} \text { value } \\
(9: 3: 3: 1)\end{array}$} & \multirow{2}{*}{$\mathrm{P}$} \\
\hline KTI & Lectin & Observed & Expected & & \\
\hline+ & + & 69 & 74.25 & \multirow{4}{*}{2.87} & \multirow{4}{*}{$0.5-0.1$} \\
\hline+ & - & 32 & 24.75 & & \\
\hline- & + & 22 & 24.75 & & \\
\hline- & - & 9 & 8.25 & & \\
\hline
\end{tabular}

+: presence, -: absence

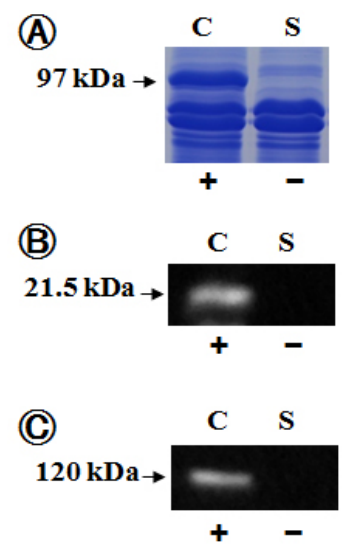

Fig. 2 Verification of the absence of lipoxygenase protein (A), Kunitz Trypsin Inhibitor (KTI) protein (B), and lectin protein (C) in the current cultivar ("Chungja\#3") and new strain. C: "Chungja\#3" (Lx1Lx1Lx2Lx2Lx3Lx3, TiTiLeLe genotype), S: new strain (lx1lx1lx2lx2lx3lx3, titilele genotype). +, -: presence or absence of lipoxygenase, lectin, and KTI proteins, respectively

lx1lx1lx2lx2lx3lx3TitiLele and KTI and lectin proteins were observed. Lectin protein of $120 \mathrm{kDa}$ and KTI protein of $21.5 \mathrm{kDa}$ were segregated in the $\mathrm{F}_{2}$ seed generation. The data of segregation ratio for presence or absence at lectin and KTI proteins are presented in Table 2.

From $132 \mathrm{~F}_{2}$ seeds obtained, KTI protein was observed in $101 \mathrm{~F}_{2}$ seeds and was not observed in $31 \mathrm{~F}_{2}$ seeds. The segregation ratio of 3:1 was observed in the $F_{2}$ seed generation for inheritance of KTI protein $\left(\chi^{2}=0.16, \mathrm{P}=0.9\right.$ - 0.5). From $132 F_{2}$ seeds obtained, lectin was observed in $91 \mathrm{~F}_{2}$ seeds and was not observed in $41 \mathrm{~F}_{2}$ seeds. For inheritance of lectin protein, the 3:1 segregation ratio was observed in the $\mathrm{F}_{2}$ seed generation $\left(\chi^{2}=2.59, \mathrm{P}=0.5-0.1\right)$. Between $L e$ allele and $T i$ allele, segregation ratio of 9 : 3 : $3: 1$ (69 Le_Ti_: 32 lele Ti_ 22 Le titi: 9 leletiti) was observed $\left(\chi^{2}=2.87, \mathrm{P}=0.5-0.1\right)$ in the $\overline{\mathrm{F}}_{2}$ seed generation. Nine $F_{2}$ seeds with triple recessive alleles (lx1lx1lx2lx2 lx3lx3titilele) were planted and one seed was not germinated. Each $\mathrm{F}_{2}$ plant with black seed coat color and green cotyledon color was harvested. Total four $F_{2}$ plants were selected. Random $F_{3}$ seeds of each $F_{2}$ plant strain were

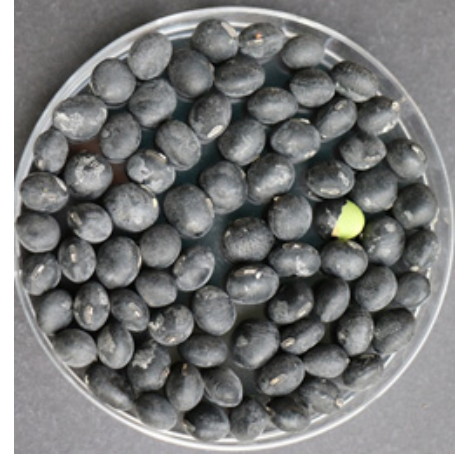

Fig. 3 Physical appearance of $\mathrm{F}_{5}$ seeds possessing triple recessive alleles (lx1lx1lx2lx2lx3lx3, titilele) expressing black seed coat and green cotyledon, with the absence of lipoxygenase, lectin, and KTI proteins

planted to advance $F_{3}$ plant generation. One $F_{3}$ plant line possessing a proper agronomical traits among four strains was selected and was harvested. Random $\mathrm{F}_{4}$ seeds were used to identify the free for lipoxygenase, lectin, and KTI proteins (Fig. 2).

The absence for lipoxygenase, lectin, and KTI proteins was confirmed in protein extracted from random $\mathrm{F}_{4}$ seeds of new strain. However, lipoxygenase, lectin, and KTI proteins were observed in the seed of "Chungja\#3" ( $L x 1$ Lx1Lx2Lx2Lx3Lx3TiTiLeLe genotype) cultivar (Fig. 2). Color of seed coat, hilum, and cotyledon was recorded on $\mathrm{F}_{5}$ seed. Seed weight ( $g / 100$ seeds) was recorded on the $F_{4}$ plant generation. $\mathrm{F}_{5}$ seeds harvested from $\mathrm{F}_{4}$ plant strain with triple null alleles (lx1lx1lx2lx2lx3lx3titilele) are shown in Figure 3. Color of seed coat for new strain was black and color of cotyledon in mature seed was green. The 100 -seed weight $(\mathrm{g})$ of new strain was 35.4.

\section{Discussion}

Soybean seeds contain $40 \%$ protein, $20 \%$ oil, $30 \%$ carbohydrate, anthocyanin, saponin, and many other nutrients to human food and animal feed. By high quantity and quality of soybean protein, demand of soybean and soybean products has increased in recent years. However, a few antinutritional factors and allergenic proteins are exist in the raw mature soybean. Lipoxygenase protein, lectin protein, and Kunitz Trypsin Inhibitor (KTI) protein are major antinutrients affecting in reducing functional or nutritional value of unprocessed soybean. To denature the activity of these antinutritional components, heating step is necessary. However, excessive heat process may lower amino acid availability of soybean and soybean products. The genetic elimination of these antinutritional components could be 
an alternative to the severe heat process. From the cross of new parent1 (lx1lx2lx3/lx1lx2lx3, tilti, Le/Le) and new parent2 (lxllx2lx3/lx1lx2lx3, Ti/Ti, le/le), $132 \mathrm{~F}_{2}$ seeds were obtained to develop a new soybean line with black seed coat color, green cotyledon color, and triple recessive alleles for lipoxygenase, lectin, and KTI proteins. The ratios of segregation for presence and absence of lectin and KTI proteins are presented in Table 2. A 3:1 segregation ratio was observed $\left(\chi^{2}=0.16, \mathrm{P}=0.9-0.5\right)$ for the presence or absence of KTI protein in the $F_{2}$ seed generation. Many researchers observed that the presence or absence of KTI protein is controlled by a single gene (Choi et al. 2016; Eun et al. 2012; Kim et al. 2006; Orf and Hymowitz, 1979). Also, a 3:1 segregation ratio was observed $\left(\chi^{2}=\right.$ $2.59, \mathrm{P}=0.5-0.1$ ) for the presence or absence of lectin protein in the $F_{2}$ seed generation. This result substantiate previous observations that lectin protein is controlled by a single gene (Choi et al. 2016; Orf and Hymowitz, 1979; Sung et al. 2013).

Between $L e$ gene and $T i$ gene, segregation ratio of 9 : $3: 3: 1$ (69 Le_Ti_ 32 lele Ti_ 22 Le_titi: 9 leletiti) was observed $\left(\chi^{2}=2.87, \mathrm{P}=0.5-0.1\right)$ in the $\mathrm{F}_{2}$ seed generation. This result agreed with previous papers that both $T i$ and $L e$ alleles were independently inherited (Choi et al. 2016; Lee et al. 2008; Moraes et al. 2006; Orf and Hymowitz, 1979). Independent inheritance of $T i$ and $L e$ loci was observed in $\mathrm{F}_{2}$ population consisted with 24 plants (Moraes et al. 2006). Orf and Hymowitz (1979) reported that Le and $T i$ alleles were inherited independently by using $\mathrm{F}_{2}$ population with 96 plants. Lee et al. (2008) reported that $\mathrm{Ti}$ and $\mathrm{Le}$ alleles were independently inherited in $173 \mathrm{~F}_{2}$ seed generation. Also, Choi et al. (2016) observed that Le and $T i$ alleles were independently inherited in $\mathrm{F}_{2}$ seed generation consisted with 179 seeds.

Nine $\mathrm{F}_{2}$ seeds with triple recessive alleles (lx1lx1lx2lx2 lx3lx3titilele genotype) from $132 \mathrm{~F}_{2}$ seeds were selected. One $F_{3}$ plant line possessing a proper agronomical traits among four strains was obtained. Random $\mathrm{F}_{4}$ seeds were used to identify the presence or absence for lipoxygenase, lectin, and KTI proteins (Fig. 2). The absence for lipoxygenase, lectin, and KTI proteins was confirmed at the mature $\mathrm{F}_{4}$ seeds of new strain. However, in the seed of "Chungja\#3" (Lx1Lx1Lx2Lx2Lx3Lx3 TiTiLeLe genotype) cultivar, lipoxygenase, KTI, and lectin proteins were observed (Fig. 2). $F_{5}$ seeds with triple recessive alleles (lx1lx1lx2lx2lx3lx3titilele) are shown in Figure 3. Color of seed coat for new strain was black and color of cotyledon was green in mature seed. The 100 -seed weight $(\mathrm{g})$ for new strain was 35.4. This is the first new black soybean strain with lx1lx1lx2lx2lx3lx3titilele genotype (absence of lipoxygenase, lectin, and KTI proteins). The strain improved newly in this study will be used to develop new soybean cultivar with black seed coat, green cotyledon, lipoxygenase protein free, KTI protein free, lectin protein free, and high quality.

\section{Acknowledgements}

This research was supported by Basic Science Research Program through the National Research Foundation of Korea (NRF) funded by the Ministry of Education (NRF2018R1D1A1B07045483).

\section{Reference}

Burns J, Gardner PT, O’Neil J, Crawford S, Morecroft I, McPhail DB, et al. (2000) Relationship among antioxidant activity, vasodilation capacity and phenolic contents of red wine. J. Agric. Food Chem. 48:220-230

Choi SW, Han SJ, Sung MK, Chung JI (2016) Breeding of black soybean line with $t i$ and le allele. Plant Breed. Biotech. 4(2):170-175

Choung MG, Baek IY, Kang ST, Han WY, Shin DC, Moon HP, et al. (2001) Isolation and determination of anthocyanins in seed coats of black soybean (Glycine max (L.) Merr.). J. Agric. Food Chem. 49:5848-5851

Chung JI (2009) A new cultivar "Gaechuck\#1": black soybean cultivar with lipoxygenase2,3-free, Kunitz trypsin inhibitor-free and green cotyledon. Korean J. Breed. Sci. 41(4):603-606

Cregan PB, Jarvik T, Bush AL, Shoemaker RC, Lark KG, Kahler AL, et al. (1999) An integrated genetic linkage map of the soybean genome. Crop Sci. 39:1464-1490

Davies CS, Nielsen SS (1986) Genetic analysis of a null-allele for lipoxygenase -2 in soybean. Crop Sci. 26:460-462

Eun HH, Sung MK, Baek WJ, Shim SI, Kim MC, Chung JI (2012) Inheritance of Kunitz trypsin inhibitor and P34 protein in soybean seed. Korean J. Crop Sci. 57(1):78-82

George M, Bhide S, Thengane R, Hosseini G, Manjaya J (2008) Identification of low lectin mutants in soybean. Plant Breed 127:150-153

Hidebrand DF, Orf JH, Hymowitz T (1980) Inheritance of an acid phosphatase and its linkage with the Kunitz trypsin inhibitor in seed protein of soybeans. Crop Sci. 20:83-85

Hildebrand DF, Hymowitz T (1981) Soybeans lacking lipoxygenase. J. Am. oil Chem. Soc. 58:583-586

Hymowitz T, Hadley HH (1972) Inheritance of a trypsin inhibitor variant in seed protein of soybeans. Crop Sci. 12:197-198

Kiang YT (1987) Mapping three protein loci on a soybean chromosome. Crop Sci. 27:44-46

Kitamura K, Davies CS, Kaizuma N, Nielsen NC (1983) Genetic 
analysis of a null-allele for lipoxygenase-3 in soybean seeds. Crop sci. 58:583-586

Kim HJ, Tsoy I, Park JM (2006) Anthocyanins from soybean seed coat inhibit the expression of TNF-alpha-induced genes associated with ischemia /reperfusion in endothelial cell by NF-kappaB-dependent pathway and reduce rat myocardial damages incurred by ischemia and reperfusion in vivo. FEBS Lett. 580:1391-1397

Kim SD, Kim YH, Park KY, Yun HT, Lee SH, Lee YH, et al. (1997) A new beany tasteless soybean variety "Jinpumkong 2" with good quality. Korean J. Breed. 29(4):502

Kobayashi AT, Hirata N, Kubota K, Kitamura (1995) Aroma constituents of soybean milk lacking lipoxygenase isozymes. J Agri Food Chem 43:2449-2452

Kunitz M (1945) Crystallization of a soybean trypsin inhibitor from soybean. Science 101:668-669

Lee KJ, Park MS, Sung MK, Kim MS, Chung JI (2008) Inheritance between $\mathrm{Le}$ gene and $\mathrm{Ti}$ gene in soybean (Glycine max L.). Korean J. Breed Sci. 40(2):97-100

MaCleod G, Ames J (1988) Soya flavor and its improvement. CRC Crit Rev Food Sci Nutr. 27:219-400

Moraes RMA, Soares TCB, Colombo LR, Salla MFS, Barros JGA, Piovesan ND, et al. (2006) Assisted selection by specific DNA markers for genetic limination of the kunitz trypsin inhibitor and lectin in soybean seeds Euphytica 149:221-226

Orf JH, Hymowitz T, Pull SP, Pueppke SG (1978) Inheritance of a soybean seed lectin. Crop Sci. 18:899-900

Orf JH, Hymowitz T (1979) Inheritance of the absence of the Kunitz trypsin inhibitor in seed protein of soybeans. Crop Sci. 19:107-109

Orf JH, Hymowitz T (1979) Soybean linkage test between Ti and Le seed proteins. Soybean Genetics Newsletter Vol. 6:32

Pull SP, Pueppke SG, Hymowitz H, Orf JH (1978) Soybean lines lacking the 120,000 daltons seed lectin. Science 200:1277-1279

Schulze H, Saini HS, Huisman J, Hessing M, Berg W, Verstegen MWA (1995) Increased nitrogen secretion by inclusion of soya lectin in the diets of pigs. J. of Science Food and Agriculture 69:501-510

Schmidt MA, Hymowitz T, Herman EM (2015) Breeding and characterrization of soybean triple null; a stack of recessive alleles of Kunitz Trypsin Inhibiotr, Soybean Agglutinin, and P34 allergen nulls. Plant Breeding. 134:310-315

Singh L, Wilson CM, Hadley HH (1969) Genetic differences in soybean trypsin inhibitors separated by disc electrophoresis. Crop Sci. 9:489-491

Sung MK, Kim MH, Seo HJ, Chung JI (2013) Inheritance of $d l m$ and $t i$ genes in soybean. Plant Breed. Biotech. 1(1):9-13 